\title{
The Hair Shedding Visual Scale: A Quick Tool to Assess Hair Loss in Women
}

\author{
María Abril Martínez-Velasco • Norma Elizabeth Vázquez-Herrera · Austin John Maddy • \\ Daniel Asz-Sigall $\cdot$ Antonella Tosti
}

Received: October 15, 2016/ Published online: February 20, 2017

(C) The Author(s) 2017. This article is published with open access at Springerlink.com

\begin{abstract}
Introduction: Hair shedding is a common consequence of the normal hair cycle that changes with internal and external factors. Female pattern hair loss (FPHL) is difficult to assess in terms of shedding severity as the conscious perception of hair shedding varies according to each individual, and most utilized methods are semi-invasive or very time consuming. In this study, we establish and validate a hair-shedding scale for women with thick hair of different lengths.
\end{abstract}

Enhanced content To view enhanced content for this article go to http://www.medengine.com/Redeem/ 5967F0600328B048.

M. A. Martínez-Velasco · D. Asz-Sigall

Universidad Nacional Autonoma de Mexico Clínica de Oncodermatología, Circuito Escolar S/N, Col. UNAM C.U., Del Coyoacán, Mexico City, Mexico

N. E. Vázquez-Herrera

Tecnologico de Monterrey, Campus Monterrey, Ave. Eugenio Garza Sada 2501, Monterrey, NL, Mexico

A. J. Maddy · A. Tosti ( $\square)$

Department of Dermatology and Cutaneous Surgery, University of Miami Miller School of Medicine, 1475 NW 12th Avenue Suite 2175, Miami, FL, USA

e-mail: atosti@med.miami.edu
Methods: A visual analog scale was developed for thick hair of short, medium, and long lengths by dividing a bundle of hairs of each length into nine piles of increasing hair amount that were then photographed and arranged in order of size. Twenty women with no FPHL with each length of hair (60 total) were asked to select the photographed hair bundle that best correlated with the amount of hair they shed on an average day. A total of 94 women with FPHL with excessive shedding were then asked to repeat the same process.

Results: Women with no FPHL and short, medium and long hair had mean shedding scores of 2.5, 2.35 and 2.4, respectively. Women with FPHL and short, medium and long hair had mean shedding scores of 7.25, 7.0 and 7.14, respectively. Statistically significant Spearman's $\rho$ coefficient and $\kappa$ coefficient demonstrated correlation and inter-observer reliability.

Conclusion: Our results show that women with FPHL not only shed considerable hair more than women with no FPHL, but that this hair-shedding visual scale is a fast and effective method of evaluating hair-shedding amounts in an office setting.

Keywords: Alopecia; Female pattern hair loss; FPHL; Hair loss; Hair shedding; Scale; Shedding; Telogen effluvium; Thick hair; Visual analogue scale 


\section{INTRODUCTION}

The hair cycle consists of multiple phases: anagen (the growing phase), catagen (the regression phase), telogen (the resting phase), exogen (the shedding phase) and kenogen (the empty phase) [1-3]. The anagen phase lasts from 2 to 6 years and is the phase of active growth that determines the length of the hair. While each follicle is in its own stage of the cycle at any given time, most scalp hairs are in the anagen phase in the normal hair cycle. After anagen, the hair follicle enters the transitional catagen phase as it shrinks and detaches from the dermal papilla. The hair then rests in the telogen phase for about 3 months until it is eventually released and shed as the new hair continues to grow during exogen. The kenogen phase indicates the period of the hair cycle in which the hair follicle remains empty after the telogen hair has shed and before a new anagen hair emerges $[4,5]$. Although the kenogen phase is observed in normal scalp, its duration and frequency are increased in androgenetic alopecia [6].

Hair shedding is, therefore, a common consequence of the normal hair cycle. The amount of hairs that are shed depends on several internal and external factors, and the conscious perception of hair shedding can vary by individual. It is logical to think that the amount of shedding should also depend on the total hair density and that women with thin hair and reduced hair density, as for instance women with advanced female pattern hair loss (FPHL), shed less than women with early FPHL or women with normal hair density, whether or not active hair loss is taking place. However, there are no specific studies on hair shedding in women with severe FPHL. Differentiating between normal hair shedding and that which is excessive can be challenging, as most patients complain that they shed more than normal, but the doctor rarely sees the amount that is shed. Common diseases causing hair loss in women include FPHL, acute and chronic telogen effluvium, alopecia areata, anagen effluvium, and cicatricial alopecias.

Female pattern hair loss is the most common disorder associated with hair shedding and is more prevalent with increasing age [7-10]. The term
FPHL is preferred to androgenetic alopecia as the majority of women with FPHL do not have increased levels of male hormones, nor other signs of increased androgen effect, and do not respond to anti-androgens with dramatic hair regrowth [11].

The pathogenesis of FPHL involves miniaturization and shortened anagen duration leading to an increase in hair shedding. Patients with FPHL may consult a doctor because they notice hair thinning, particularly over the mid-frontal scalp or because they notice increased hair shedding. In fact, FPHL is often precipitated and exacerbated by conditions that cause telogen effluvium, such as drugs, acute stressors, weight loss, and partum. Sinclair et al. found that $60 \%$ of women presenting with increased hair shedding, without any discernible thinning or reduction in hair density, had androgenetic alopecia on scalp biopsy [12].

Early diagnosis of FPHL is very important in order to prevent disease progression while miniaturization is still in the initial phases and possibly reversible. This can be achieved by dermoscopy, which is very helpful in diagnosing early disease.

An important distinguishing feature of FPHL on dermoscopy is the presence of hairs with different thickness (hair diameter diversity), which reflect the miniaturization process $[13,14]$. Another typical dermoscopic feature of FPHL is the presence of more than six thin short regrowing hairs in the frontal scalp. Dermoscopy also distinguishes FPHL from chronic telogen effluvium (CTE), another disorder characterized by increased shedding and loss of volume that is often difficult to assess with objective methods.

The pull test is the most utilized test to assess hair shedding in clinical practice [15]; however, it is not very sensitive and most women with FPHL or CTE have a negative pull test even if they complain of excessive shedding. Other more precise methods to assess hair shedding are either semi-invasive (trichogram, phototrichogram) or very time consuming (wash test, modified wash test) and difficult to utilize in the office $[16,17]$. A visual tool that could quickly provide an idea of the hair shedding amount in an office setting would aid the doctor's and patient's assessment of hair loss. Two recent 
papers proposed and evaluated the efficacy of a visual scale for evaluating hair shedding of women. However, these scales were made for women with thin blond straight hair $[18,19]$. The aim of this study is to establish and validate a hair-shedding scale for women with thick curly hair of different lengths.

\section{METHODS}

All procedures followed were in accordance with the ethical standards of the responsible committee on human experimentation (institutional and national) and with the Helsinki Declaration of 1964, as revised in 2013. Informed consent was obtained from all patients for being included in the study.

A visual scale was developed for thick curly hair of each hair length (shoulder, mid-back, and lower back) by dividing a bundle of hairs of each length into nine piles of increasing hair amount (10, 50, 100, 200, 300, 400, 500, 600, and 700 hairs) (Figs. 1, 2, 3). Each pile was photographed and arranged in order of size. The hairs were measured in thickness as well as length. Due to the elliptical shape of hair, in order to obtain a value of hair thickness the cross-sectional area of the hairs was calculated. Vivosight by Michelson Diagnostics Optical Coherence Tomography was used to measure the maximal and minimal diameters of the hairs. Since hair has different thickness along the shaft, proximal (A), medial (B), and distal (C) levels were considered. The mean value for A, B and C was used for this study.

Shoulder length hair had average thickness of $0.078 \mathrm{~mm}$ and length of $24.0 \mathrm{~cm}$, medium length hair had average thickness of $0.094 \mathrm{~mm}$ and length of $32.5 \mathrm{~cm}$, while long lower back hair had average thickness $1.06 \mathrm{~mm}$ and length of $52.3 \mathrm{~cm}$.

Hair curliness was evaluated using the visual scale proposed by Loussouarn et al., which defined hair curliness on a scale of I-VIII based on simple measurements using curve diameter meters and rulers [20]. The hairs utilized to create the scale in our study were graded as type III for all three different lengths.

To validate the scale, we showed a picture with the scale to 20 asymptomatic women with shoulder length hair, 20 asymptomatic women with medium length hair, and 20 asymptomatic women with lower back length hair. They were asked to look at the nine photos and select the photograph that best correlated with the amount of hair they shed when brushing or combing on an average shampooing day. Subjects were asked to reevaluate their score $48 \mathrm{~h}$ later.

We also validated the scale in 94 women with FPHL complaining of excessive shedding. None of these patients had been treated before, and diagnosis of FPHL was performed by clinical examination and dermoscopy. Dermoscopic pictures were taken in three scalp areas after central parting (vertex, middle and frontal scalp) at $\times 20$ and $\times 40$ magnification. FPHL was diagnosed based on the presence of two validated dermoscopic criteria: more than 20\% variability in the hair shaft diameter, and the presence of more than seven short regrowing hairs in the frontal scalp [21, 22]. These included 34 patients with shoulder length curly hair, 31 with medium length curly hair, and 29 with long curly hair. Patients were asked to score their hair loss using the scale on their first visit, as well as 1 week later. Patients were consecutively recruited during a 5-month period.

To validate if patient's perception of shedding from the scale correlated with the actual hairs shed, we manually counted the hairs from two additional women with medium length hair affected by FPHL and excessive shedding, who were not part of the original study. These patients were asked to shampoo every other day for 2 weeks and to bring the hairs shed during each shampoo in separate bags after grading the amount of shedding using the scale.

\section{RESULTS}

Results of the shedding scores for normal women not complaining of hair shedding and women with FPHL complaining of excessive shedding are summarized in Tables 1 and 2.

\section{Normal Women (Table 1)}

Normal women with shoulder hair had a mean shedding score of 2.50, Spearman's $\rho$ coefficient 

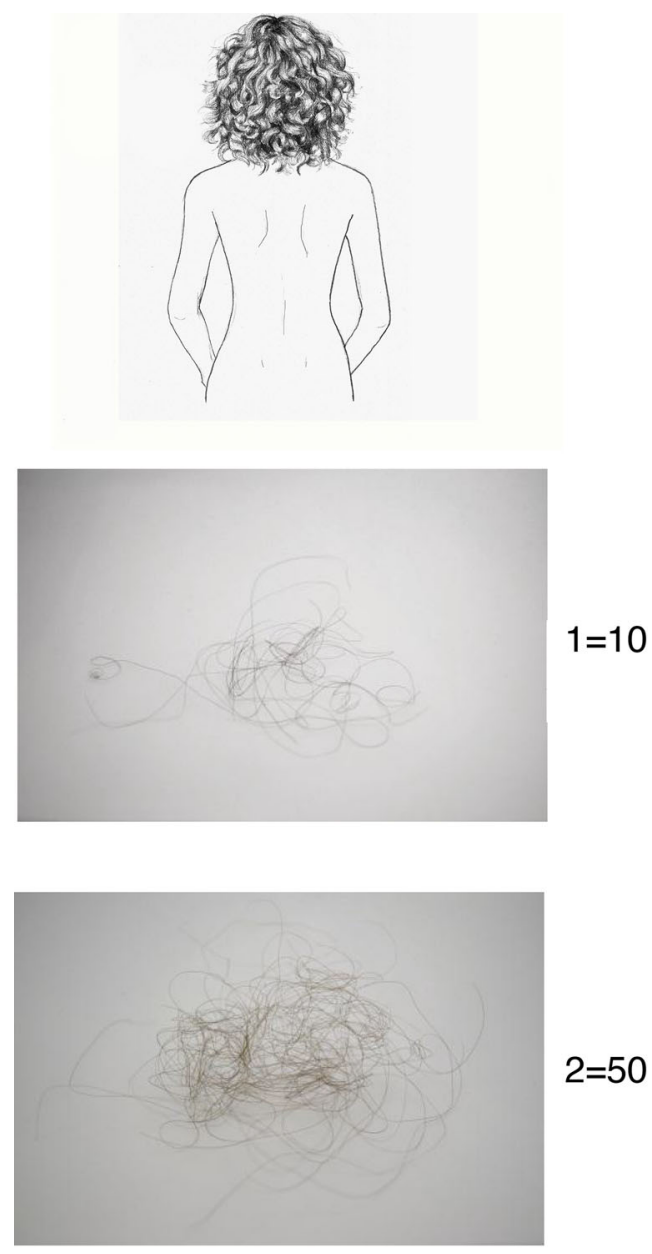

$2=50$

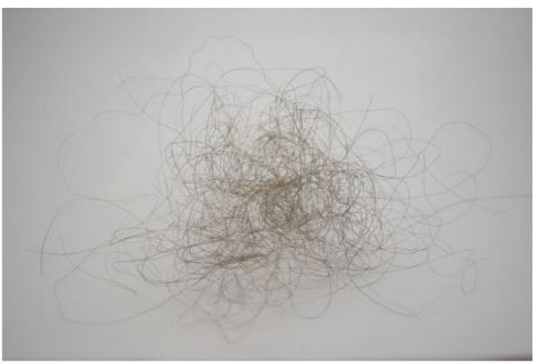

$3=100$

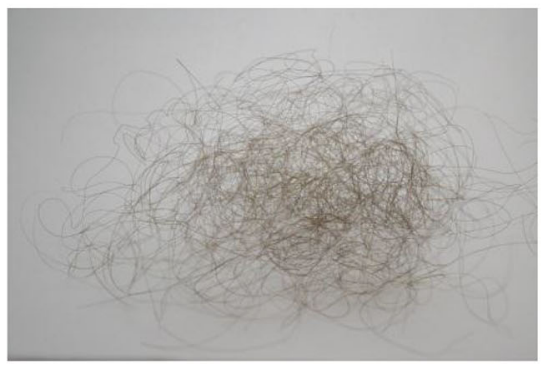

$4=200$

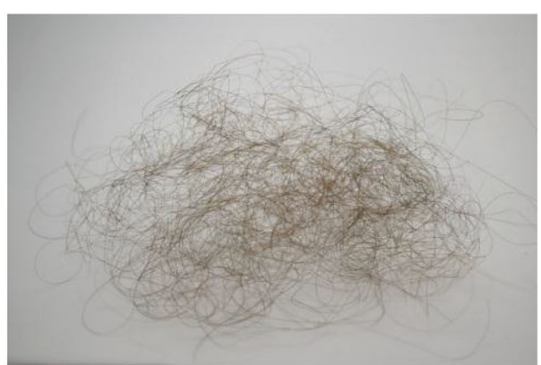

$5=300$

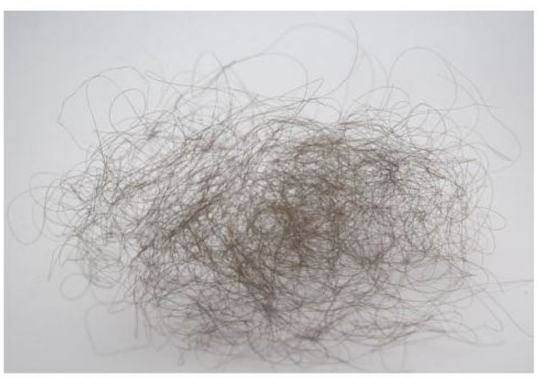

$6=400$

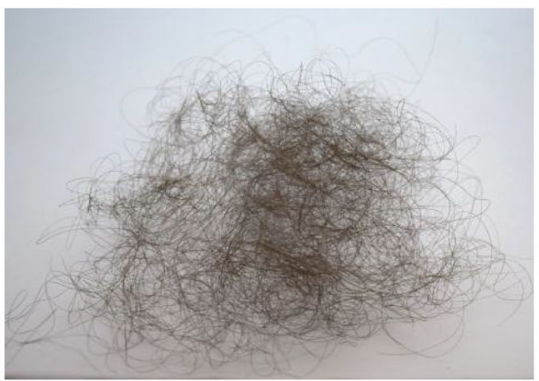

$7=500$

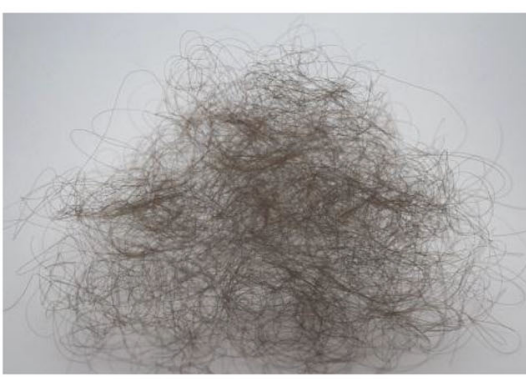

$8=600$

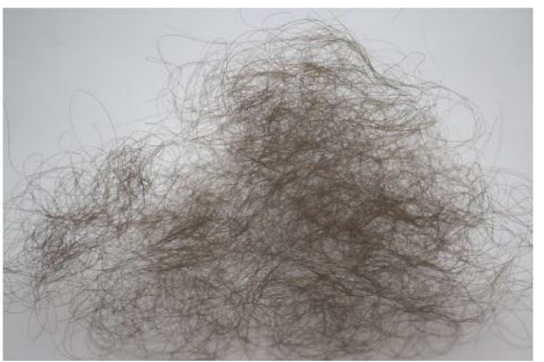

$9=700$

Fig. 1 Shedding scale short length hair (1:1) 

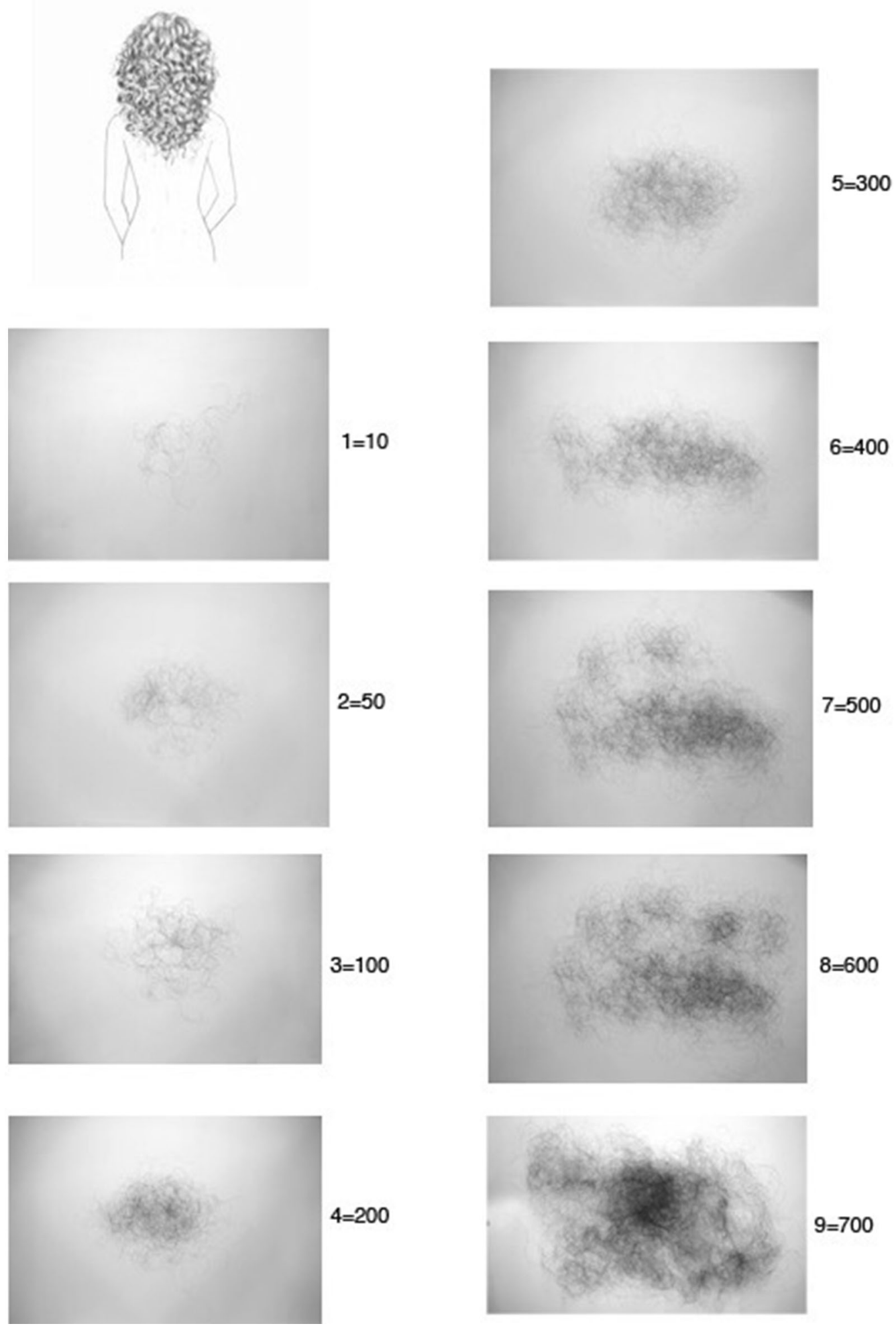

Fig. 2 Shedding scale medium length hair (1:1) 

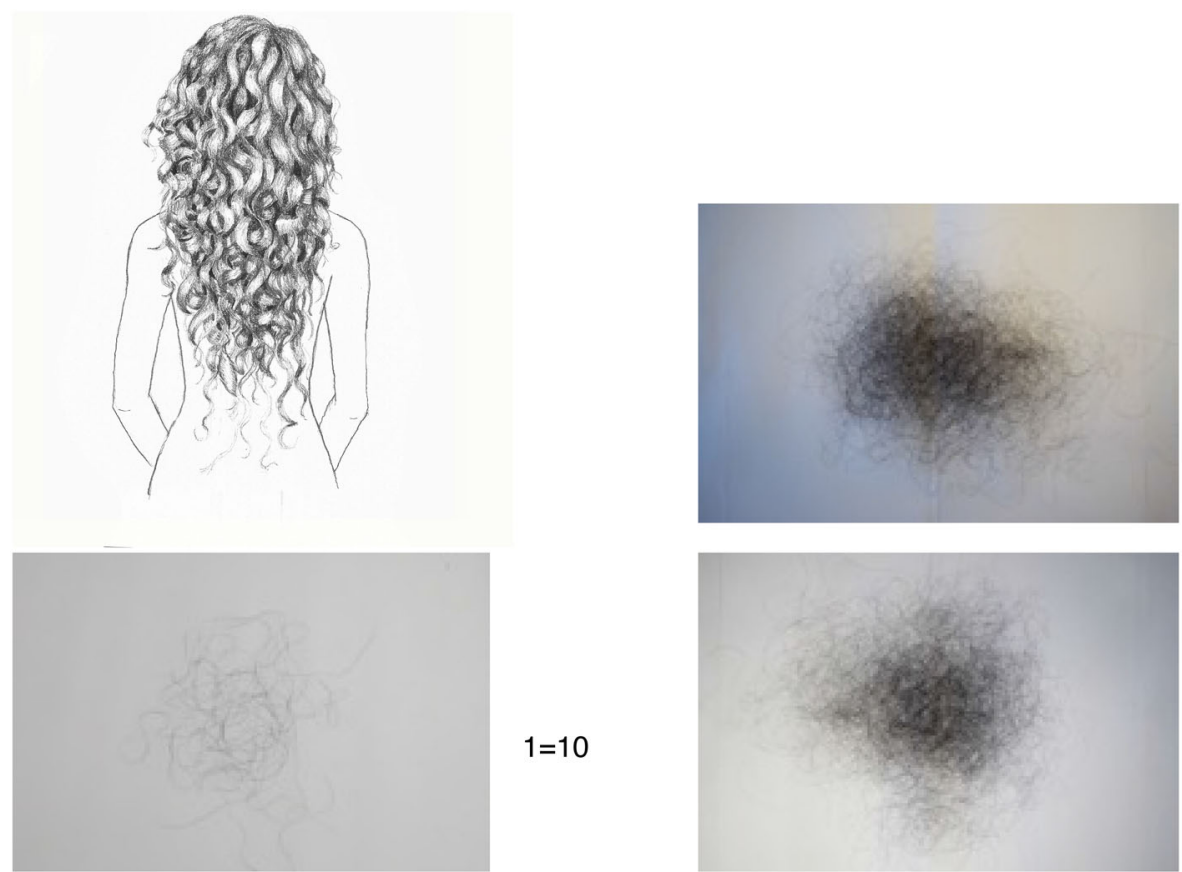

$5=300$
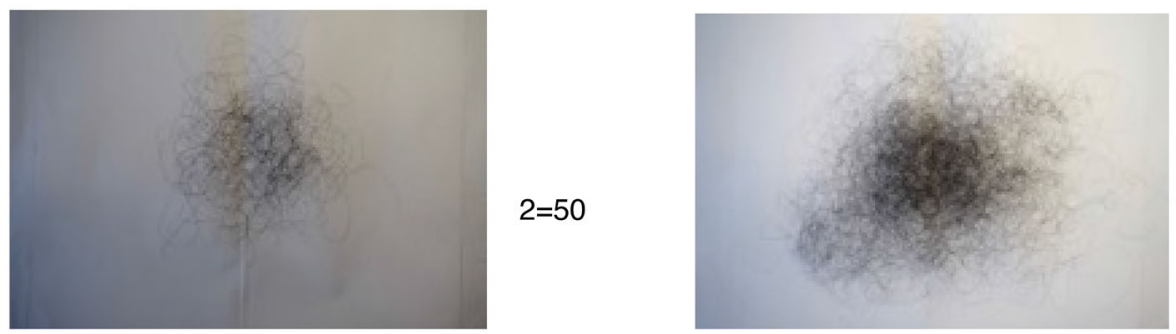

$6=400$
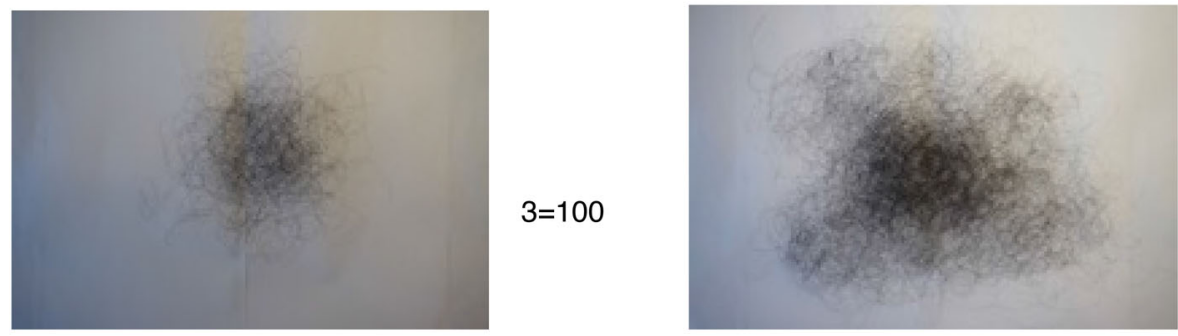

$7=500$
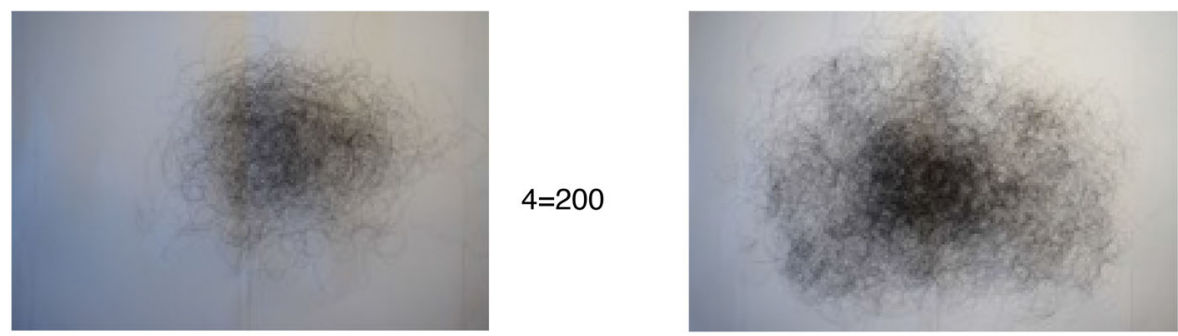

Fig. 3 Shedding scale long hair (1:1) 
Table 1 Hair-shedding scale results, healthy women

\begin{tabular}{|c|c|c|c|c|c|c|}
\hline \multirow[t]{3}{*}{ Hair-shedding score } & \multicolumn{6}{|c|}{ Number of patients that chose the corresponding hair-shedding score } \\
\hline & \multicolumn{2}{|c|}{ Short hair } & \multicolumn{2}{|c|}{ Medium-length hair } & \multicolumn{2}{|l|}{ Long hair } \\
\hline & 1st visit & 2nd visit & 1st visit & 2nd visit & 1st visit & 2nd visit \\
\hline \multicolumn{7}{|c|}{ Hair-shedding score in patients without FPHL } \\
\hline 1 & 2 & 2 & 5 & 5 & 3 & 3 \\
\hline 2 & 9 & 8 & 6 & 6 & 8 & 7 \\
\hline 3 & 6 & 7 & 6 & 7 & 7 & 8 \\
\hline 4 & 3 & 3 & 3 & 2 & 2 & 2 \\
\hline 5 & 0 & 0 & 0 & 0 & 0 & 0 \\
\hline 6 & 0 & 0 & 0 & 0 & 0 & 0 \\
\hline 7 & 0 & 0 & 0 & 0 & 0 & 0 \\
\hline 8 & 0 & 0 & 0 & 0 & 0 & 0 \\
\hline 9 & 0 & 0 & 0 & 0 & 0 & 0 \\
\hline Total & 20 & & 20 & & 20 & \\
\hline Mean $\pm S D$ & $2.50 \pm 0.8$ & $2.55 \pm 0.8$ & $2.35 \pm 1.0$ & $2.40 \pm 0.9$ & $2.40 \pm 0.8$ & $2.45 \pm 0.8$ \\
\hline$\rho$ Spearman & 0.955 & & $<0.978$ & & 0.956 & \\
\hline$p$ value & $<0.0001$ & & $<0.0001$ & & $<0.0001$ & \\
\hline$\kappa$ & 0.927 & & 0.932 & & 0.927 & \\
\hline$p$ value & $<0.0001$ & & $<0.0001$ & & $<0.0001$ & \\
\hline
\end{tabular}

Hair-shedding score: 1-4 normal hair shedding, 5-9 excessive hair shedding

$0.955(p<0.0001)$ and $\kappa 0.927 \quad(p<0.0001)$. Normal women with medium length hair had a mean shedding score of 2.35, Spearman's $\rho$ coefficient $0.978 \quad(p<0.0001)$ and $\kappa \quad 0.932$ $(p<0.0001)$. Normal women with long hair had a mean shedding score of 2.40, Spearman's $\rho$ coefficient $0.956 \quad(p<0.0001)$ and $\kappa \quad 0.927$ $(p<0.0001) . \kappa$ was excellent in each of the groups of normal women.

\section{Female Pattern Hair Loss (Table 2)}

Women with FPHL and shoulder hair had a mean shedding score of 7.25, Spearman's $\rho$ coefficient $0.986 \quad(p<0.0001)$ and $\kappa 0.963$ $(p<0.0001)$. Data are reported in detail in Table 2. Women with FPHL and medium length hair had a mean shedding score of 7.0, Spearman's $\rho$ coefficient $0.994 \quad(p<0.0001)$ and $\kappa 0.920(p<0.0001)$. Women with FPHL and long hair had a mean shedding score of 7.14, Spearman's $\rho$ coefficient $0.990(p<0.0001)$ and $\kappa 0.956(p<0.0001)$.

\section{Manual Hair Count}

Table 3 reports the results of the manual hair count of 15 samples. Statistical analysis showed strong correlation between shedding score and manual counting. Pearson correlation 0.88, $p<0.05$.

\section{DISCUSSION}

Female patients with hair loss of different causes frequently come to the office with pictures or even bags of hairs to document their problem. It 
Table 2 Hair-shedding scale results, FPHL women

\begin{tabular}{|c|c|c|c|c|c|c|}
\hline \multirow[t]{3}{*}{ Hair-shedding score } & \multicolumn{6}{|c|}{ Number of patients that chose the corresponding hair-shedding score } \\
\hline & \multicolumn{2}{|l|}{ Short hair } & \multicolumn{2}{|c|}{ Medium-length hair } & \multicolumn{2}{|l|}{ Long hair } \\
\hline & 1st visit & 2nd visit & 1st visit & 2nd visit & 1st visit & 2nd visit \\
\hline \multicolumn{7}{|c|}{ Hair-shedding score FPHL patients } \\
\hline 1 & 0 & 0 & 1 & 1 & 0 & 0 \\
\hline 2 & 1 & 1 & 1 & 1 & 2 & 2 \\
\hline 3 & 1 & 1 & 0 & 0 & 1 & 1 \\
\hline 4 & 1 & 1 & 1 & 1 & 1 & 1 \\
\hline 5 & 2 & 2 & 2 & 2 & 1 & 1 \\
\hline 6 & 3 & 3 & 4 & 4 & 2 & 2 \\
\hline 7 & 7 & 7 & 5 & 6 & 6 & 7 \\
\hline 8 & 10 & 9 & 7 & 8 & 7 & 6 \\
\hline 9 & 9 & 10 & 8 & 8 & 9 & 9 \\
\hline Total & 34 & & 31 & & 29 & \\
\hline Mean \pm SD & $7.25 \pm 1.7$ & $7.29 \pm 1.8$ & $7.0 \pm 2.0$ & $7.06 \pm 2.0$ & $7.14 \pm 2.1$ & $7.10 \pm 2.0$ \\
\hline$\rho$ Spearman & 0.986 & & 0.994 & & 0.990 & \\
\hline$p$ value & $<0.0001$ & & $<0.0001$ & & $<0.0001$ & \\
\hline$\kappa$ & 0.963 & & 0.920 & & 0.956 & \\
\hline$p$ value & $<0.0001$ & & $<0.0001$ & & $<0.0001$ & \\
\hline
\end{tabular}

Hair-shedding score: 1-4 normal hair shedding, 5-9 excessive hair shedding

is, therefore, important for dermatologists who are not specialized in hair disorders to have a fast tool that allows them to distinguish normal from abnormal. The hair-shedding scale is, in our opinion, the best instrument to feed this need. In fact, the time required to explain the scale to the patient and to obtain the patient's perception of shedding by looking at the scale was always within 2 min.

The hair-shedding scale is not a diagnostic instrument, as excessive hair shedding can be a symptom of numerous hair disorders, but it is a tool that helps in discriminating normal from excessive shedding in a busy office practice. It should be utilized together with other objective diagnostic methods, particularly trichoscopy, which are essential in identifying early FPHL. Although not validated in the present study, the scale could also allow doctors to roughly establish the amount of hair that is shed when patients bring bags of hair to the office. Frequency of shampooing and brushing habits can definitely influence the amount of shedding, and should always be considered when evaluating results.

Our hair-shedding scale is designed for Caucasian women with thick hair, and the thickness of the hair fibers utilized to create the scale correlate well with the reported thickness of Caucasian thick hair [23]. It well complements the scale that has already been validated for Caucasian women with blond thin hair. A different scale needs to be created for women of Asian or African ethnicity.

This hair-shedding visual scale showed an excellent observable correlation and 
Table 3 Correlations between scale results and manual counting in 14 samples from 2 women

\begin{tabular}{llll}
\hline Day & Visual scale & Manual count & \\
\hline 1 & $5(300)$ & 275 & Patient 1 \\
2 & $6(400)$ & 380 & \\
3 & $5(300)$ & 307 & \\
4 & $5(300)$ & 296 & \\
5 & $7(500)$ & 435 & \\
6 & $6(400)$ & 366 & \\
7 & $5(300)$ & 271 & \\
8 & $5(300)$ & 310 & \\
1 & $4(200)$ & 163 & \\
2 & $6(400)$ & 432 & \\
3 & $5(300)$ & 330 & \\
4 & $3(100)$ & 215 & \\
5 & $6(400)$ & 382 & \\
6 & $5(300)$ & 315 & \\
7 & $5(300)$ & 367 & \\
Pearson & 0.88 & & \\
$p$ value & $<0.05$ & & \\
\hline
\end{tabular}

The visual scale is indicated as a numerical grade followed by numerical value in parenthesis

concordance in both healthy women and women with excessive hair shedding. Women with no FPHL had a statistically significant lower score of hair shedding in the visual scale than women with FPHL. All healthy women had a score of $1-4$, which would be considered as normal hair shedding. Mean values were within 2 and 3, showing that most women not complaining of hair loss graded their shedding as less than 100 hairs.

For women with FPHL, 91\% of women with shoulder hair, $90 \%$ of women with medium-length hair and $86 \%$ of women with long hair chose images 5-9 which correspond to excessive hair shedding. These results are in accordance with the results of a recent study on the prevalence of hair shedding in females not suffering from FPHL using the published visual analog scale. This study found that $60 \%$ of women (21 out of 37) with FPHL reported excessive hair shedding versus $40 \%$ of women (104 out of 263) without FPHL on hair washing days [19]. In this study, excessive shedding was found to be inversely proportional to age, while being strongly correlated with hair length, as most of the subjects who reported excessive shedding had hair longer than shoulder length. These results point out that a single scale is not able to accurately assess shedding and that scales for different hair length are needed.

One possible limitation of this study is that women who believe they have excess hair shedding may be more likely to choose hair bundles at the higher end of the scale compared to those who do not perceive the same extent of hair loss; however, we believe this does not change the usefulness of the scale in clinical practice. The scale should not be used as a precise measure of hair loss but instead as a tool to quickly distinguish normal from abnormal. Although we decided to provide a 9-grade scale, differences within the very abnormal ranges might not possibly be detected. However, our manual count results indicate that perception of shedding was quite accurate within grades. Other factors, particularly psychological factors such as anxious preoccupation, helplessness, and feelings of diminished attractiveness, can also drive the perception of hair loss and are known to co-occur with hair shedding in women [24].

\section{CONCLUSIONS}

The hair-shedding visual scale is a fast and effective method of evaluating hair-shedding amount as well as the patient's perception of hair shedding. Using the visual scale, doctors and patients will be able to grossly estimate hair loss severity before and after treatment. In this study, we did not evaluate the use of the scale in assessing response to treatments, but we think that the scale can become a part of the tools that we use to gauge a patient's progression with therapy. Ongoing use of this scale may be valuable in evaluating response to therapy as well as decreasing patient 
anxiety, as they will be able to objectively assess their treatment response. This scale is a quick and simple tool that can be used in an office setting and is effective in patients of all hair lengths including short, medium, and long length thick hair.

\section{ACKNOWLEDGEMENTS}

No funding or sponsorship was received for this study or publication of this article. All named authors meet the International Committee of Medical Journal Editors (ICMJE) criteria for authorship for this manuscript, take responsibility for the integrity of the work as a whole, and have given final approval for the version to be published.

Disclosures. María Abril Martínez-Velasco, Norma Elizabeth Vázquez-Herrera, Austin John Maddy, Daniel Asz-Sigall have nothing to disclose. Dr Antonella Tosti is consultant for DS laboratorios, P\&G, Fotofinder, and PI for Incyte.

Compliance with Ethics Guidelines. All procedures followed were in accordance with the ethical standards of the responsible committee on human experimentation (institutional and national) and with the Helsinki Declaration of 1964, as revised in 2013. Informed consent was obtained from all patients for being included in the study.

Data Availability. The datasets during and/ or analyzed during the current study are available from the corresponding author on reasonable request.

Open Access. This article is distributed under the terms of the Creative Commons Attribution-NonCommercial 4.0 International License (http://creativecommons.org/licenses/ by-nc/4.0/), which permits any noncommercial use, distribution, and reproduction in any medium, provided you give appropriate credit to the original author(s) and the source, provide a link to the Creative Commons license, and indicate if changes were made.

\section{REFERENCES}

1. Higgins CA, Westgate GE, Jahoda CAB. From telogen to exogen: mechanisms underlying formation and subsequent loss of the hair club fiber. J Invest Dermatol. 2009;129:2100-8.

2. Stenn K. Exogen is an active, separately controlled phase of the hair growth cycle. J Am Acad Dermatol. 2005;52:374-5.

3. Oh JW, et al. A guide to studying human hair follicle cycling in vivo. J Invest Dermatol. 2016;136:34-44.

4. Guarrera M, Rebora A. Kenogen in female androgenetic alopecia. A longitudinal study. Dermatology. 2005;210:18-20.

5. Rebora A, Guarrera M. Kenogen. A new phase of the hair cycle? Dermatology. 2002;205:108-10.

6. Courtois M, Loussouarn G, Hourseau C, Grollier JF. Hair cycle and alopecia. Skin Pharmacol. 1994;7:84-9.

7. Gan DCC, Sinclair RD. Prevalence of male and female pattern hair loss in Maryborough. J Investig Dermatol Symp Proc. 2005;10:184-9.

8. Hamilton JB. Patterned loss of hair in man; types and incidence. Ann N Y Acad Sci. 1951;53:708-28.

9. Birch MP, Messenger JF, Messenger AG. Hair density, hair diameter and the prevalence of female pattern hair loss. Br J Dermatol. 2001;144:297-304.

10. Siah T, Muir-Green L, Shapiro J. Female pattern hair loss: a retrospective study in a tertiary referral center. Int J Trichol. 2016;8:57.

11. Olsen EA. Female pattern hair loss. J Am Acad Dermatol. 2001;45:S70-80.

12. Sinclair R, Jolley D, Mallari R, Magee J. The reliability of horizontally sectioned scalp biopsies in the diagnosis of chronic diffuse telogen hair loss in women. J Am Acad Dermatol. 2004;51:189-99.

13. de Lacharrière $\mathrm{O}$, et al. Hair diameter diversity: a clinical sign reflecting the follicle miniaturization. Arch Dermatol. 2001;137:641-6.

14. Tosti A, Iorizzo M, Piraccini BM. Androgenetic alopecia in children: report of 20 cases. Br J Dermatol. 2005;152:556-9.

15. Blume-Peytavi U, Tosti A, Whiting D, Trüeb R, editors. Hair growth and disorders. Berlin: Springer; 2008. p. 125-57. doi:10.1007/978-3-540-46911-7_8. 
16. Guarrera, M. Additional methods for diagnosing alopecia and appraising their severity. G Ital Dermatol Venereol 2014;149(1):93-102.

17. Rebora A., Guarrera M, Baldari M, Vecchio F. Distinguishing Androgenetic alopecia from chronic telogen effluvium when associated in the same patient. Arch Dermatol. 2005;141(10):1243-5.

18. Sinclair R. Hair shedding in women: how much is too much? Br J Dermatol. 2015;173:846-8.

19. Kovacevic M, et al. Prevalence of hair shedding among women. Dermatol Ther. 2016;. doi:10.1111/ dth.12415.

20. Loussouarn $G$, et al. Worldwide diversity of hair curliness: a new method of assessment. Int J Dermatol. 2007;46:2-6.
21. Rakowska A, Slowinska M, Kowalska-Oledzka E, Olszewska M, Rudnicka L. Dermoscopy in female androgenic alopecia: method standardization and diagnostic criteria. Int J Trichol. 2009;1:123-30.

22. Herskovitz I, de Sousa ICVd, Tosti A. Vellus hairs in the frontal scalp in early female pattern hair loss. Int J Trichol. 2013;5:118-20.

23. Bouabbache $\mathrm{S}$, et al. What is a Caucasian 'fine' hair? Comparing instrumental measurements, self-perceptions and assessments from hair experts. Int J Cosmet Sci. 2016;38:581-8.

24. Cash TF, Price VH, Savin RC. Psychological effects of androgenetic alopecia on women: comparisons with balding men and with female control subjects. J Am Acad Dermatol. 1993;29:568-75. 